\title{
Écouter le silence ? À propos de Retour des corps, parcours des âmes. Exhumations et deuils collectifs dans le monde hispanophone
}

\section{Emma Gobin}

\section{(2) OpenEdition Journals}

Édition électronique

URL : https://journals.openedition.org/jsa/17055

DOI : 10.4000/jsa. 17055

ISSN : 1957-7842

Éditeur

Société des américanistes

Édition imprimée

Date de publication : 15 juin 2019

ISBN : 978-2-902715-11-4

ISSN : 0037-9174

\section{Référence électronique}

Emma Gobin, «Écouter le silence ? À propos de Retour des corps, parcours des âmes. Exhumations et deuils collectifs dans le monde hispanophone », Journal de la Société des américanistes [En ligne], 105-1 I 2019, mis en ligne le 15 juin 2019, consulté le 04 septembre 2022. URL : http:// journals.openedition.org/jsa/17055; DOI : https://doi.org/10.4000/jsa.17055

Ce document a été généré automatiquement le 4 septembre 2022

Tous droits réservés 


\title{
Écouter le silence? À propos de Retour des corps, parcours des âmes. Exhumations et deuils collectifs dans le monde hispanophone
}

\author{
Emma Gobin
}

\section{RÉFÉRENCE}

Écouter le silence ? À propos de Retour des corps, parcours des âmes. Exhumations et deuils collectifs dans le monde hispanophone, sous la direction de Anne-Marie Losonczy et Valérie Robin Azevedo, Éditions Pétra (« Les cadavres dans les génocides et les violences de masse »), Paris, 2016, 216 p., réf. dissém., cahier photographique de 8 p. (en noir et en coul.).

1 Rassemblant sept contributions d'anthropologues travaillant sur l'Espagne, le Pérou, le Guatemala et la Colombie, Retour des corps, parcours des âmes est un ouvrage dont le lecteur ne peut ressortir indemne. Impactant, obsédant, il submerge son lecteur dans le monde des conflits armés et de leurs " réparations », un monde jonché de cadavres et de restes humains mutilés, peuplé d'âmes en peine appartenant tant aux morts qu'aux vivants. Important par les données historiques et ethnographiques qu'il présente ${ }^{1}$, le volume l'est aussi par les questionnements qu'il soulève et dont la portée dépasse de loin les seuls terrains envisagés. Comment les sociétés contemporaines font-elles face aux situations de violence interne - répressions armées, guerres civiles, massacres - qui les ont déchirées? Quand, par qui et en quels lieux ces violences et les disparitions physiques qui en ont découlé sont-elles reconnues, mises en mots et en mémoire? Qu'en est-il des leviers et des injonctions du droit international qui, en contexte de globalisation, favorisent de tels processus et font de l'exhumation (de charniers, de fosses communes) l'une des pièces-maîtresses de dispositifs de "réconciliation » orchestrés à l'échelle nationale? Pour y répondre, le volume réunit des études de cas minutieuses 
centrées sur la thématique de cette récente collection des éditions Pétra (« Les cadavres dans les génocides et les violences de masse »)2. Ouvrant différentes voies d'analyse, toutes prennent pour point de départ l'étude de ces moments difficiles que sont les exhumations de cadavres des conflits passés, autant que les épisodes de restitution de ces restes humains violentés à leurs proches vivants.

2 Exemplaires du forensic turn ayant marqué les procédures de la «justice transitionnelle ", de telles pratiques structurent de nombreux processus de pacification en contexte de "post-conflit »; catégorie du vocabulaire des instances internationales qui, comme le relèvent avec acuité les coordinatrices du volume, indexe une temporalité ambiguë "entre guerre et paix", empreinte d'incertitude et d'une "culture de la terreur » liée aux violences passées autant qu'à la crainte de leur possible retour (p. 8, 14). L'introduction de l'ouvrage et la contribution de V.Sanford convergent ainsi pour rappeler que ces pratiques séquencées en quatre étapes (excavation, identification, dévolution, ré-enterrement [p. 19]) se sont "scientificisées » et normalisées au fil des années 1990 au point d'être désormais largement déployées de par le monde. Pour ceux qui les promeuvent (parmi eux, des instances supranationales, des États, les « commissions d'histoire » ou « de vérité » qui ont vu le jour au fil des années 1990 en Amérique latine, des associations militantes ou encore les experts médico-légaux), de telles procédures ont pour vertu de permettre d'« honorer » et de " dignifier » les morts, de reconnaître et d'adouber leur statut de "victimes ", lequel s'étend à leurs proches vivants, bénéficiaires de politiques de dédommagements symboliques et économiques (voir notamment les contributions de V. Robin-Azevedo et C. Duterme). Supposées favoriser l'exercice de la démocratie, elles sont ainsi perçues comme essentielles à l'avènement d'ordres socio-politiques nouveaux, potentiellement apaisés par l'écriture et l'intégration d'une "vérité " dont le sens et la valeur peuvent être requalifiés (par exemple à travers la catégorisation, sur des bases médico-légales, des crimes passés au rang de " génocide ", comme dans le cas du Guatemala évoqué par Sanford).

3 Envisageant cependant le vécu des populations visées par ces dispositifs, l'ouvrage interroge les tensions qui émergent des modalités concrètes d'implémentation de cet idéal de justice réparatrice sur différents terrains, lesquelles, à la croisée du global et du local, s'avèrent fréquemment achopper sur des "cosmovisions" situées et/ou des structures de domination pérennes. Comment et en quels lieux se construisent et se reconfigurent le rapport collectif à la mort et au deuil ainsi que le tissu des relations sociales ordinaires, grevé de non-dits? Comment ces dispositifs sont-ils investis ou non par les groupes concernés ? Au-delà d'une gestion de plus en plus «bureaucratique » de la douleur (p. 130), qu'en est-il du vécu de la violence, de ses formes d'expression autant que des éventuelles formes de violence qui, à leur tour, encadrent ces phénomènes? Entre espaces intimes et publics, entre échecs et points de rencontre, les auteurs parviennent alors à mettre au jour, à travers une démarche d'analyse résolument multiscalaire, la multiplicité des registres discursifs et d'action qui, sans toujours se recouvrir, opèrent à l'interface de ces groupes avec des interlocuteurs officiels « externes» ainsi que dans les méandres d'« entre-soi » communautaires (familiaux, villageois) fédérés par des dynamiques de présentation du collectif et/ou fracturés par des expériences différenciées de la violence (voir notamment la contribution de D. Delacroix).

4 Intitulée "L'intimité de la défaite: autour des exhumations dans l'Espagne contemporaine », la contribution de F. Ferrándiz Martín considère ainsi la question de la 
temporalité et de la génération à l'œuvre dans les dynamiques de "récupération de la mémoire historique » orchestrées par les petits-enfants des Républicains de la guerre civile. Interrogeant les effets du déplacement d'une mémoire intime, longtemps illégitime, «des vaincus» dans l'espace politique national, l'auteur relève combien, après des décennies de mise en silence (par l'exil et la terreur, par la transmission à demimots ou à voix basse de récits conservés dans le « secret » des familles), le processus de recherche des fosses de la guerre s'avère redessiner une cartographie inédite du territoire espagnol qui dérange les représentations collectives du passé national en même temps qu'elle s'accompagne de la production de nouveaux récits d'affliction, dans lesquels l'émotion opère à plein.

5 Le chapitre présenté par A.Cecconi ("Quand les disparus réapparaissent en rêve. Un regard comparé entre Pérou et Espagne ») évoque des récits d'expériences oniriques impliquant le dialogue avec les morts de la guerre et dont le recueil montre d'abord combien le travail de mémoire à l'œuvre est distribué, tant en termes de génération que de genre. L'analyse la conduit alors à soulever la question du statut culturel et politique du rêve. Tandis qu'au Pérou, ce dernier apparaît souvent comme un vecteur direct de l'engagement des familles dans les processus d'exhumation, selon une dynamique où ces "revenants" masculins opèrent aussi in fine comme des agents (ce sont eux qui " réclament » leur "ré-inhumation» en envahissant les nuits de celles qui leur ont survécu), il fonctionne plutôt comme un complément d'ordre symbolique et psychologique minoré en Espagne, où une telle subjectivation semble mettre en péril la récente dynamique collective de politisation de la mémoire à l'échelle nationale. Car si cet ouvrage est sensible (par les sujets qu'il aborde et par la façon dont il les aborde), il l'est aussi aux logiques de pouvoir impliquées et aux formes de reproduction de la violence que reconduisent les dispositifs déployés, destinés à des groupes souvent dominés, en partie « altérisés » au sein de la nation.

6 Fondée sur sa participation précoce aux premières exhumations menées sur les lieux du massacre de Plan de Sánchez (Guatemala), et sur son implication croissante en tant qu'experte scientifique, la contribution de V. Sanford qui s'intitule «Sur la ligne de front : l'anthropologie médico-légale » souligne ainsi comment de nouveaux espaces de citoyenneté ont été gagnés dans un contexte initial de terreur propice au statu quo («laissez les morts en paix!» intimaient alors aux familles mayas les militaires guatémaltèques qui rôdaient autour des premières excavations [p. 65]). Comme le montre l'article de V. Robin-Azevedo (« Rendre leur dignité aux disparus de la guerre? Exhumations, justice réparatrice et politique de la compassion au Pérou »), les traces de cette violence sont aussi à analyser à plusieurs niveaux aujourd'hui. Le premier touche notamment au choix de l'État péruvien de ritualiser sur une scène publique, médiatique, les dévolutions et les ré-inhumations de corps liés à des massacres soigneusement sélectionnés - dimension qui engage tout le problème de la concurrence des mémoires et de la construction en actes d'un récit qui, en la forme, occulte les exactions des uns (l'État) au profit de la stigmatisation et de la dépolitisation des violences des autres (celles de la guérilla du Sentier lumineux). Le second touche aux conditions matérielles dans lesquelles sont restitués ces restes humains à leurs proches, lesquelles renvoient les groupes paysans concernés à leur infériorité sociale, leur pauvreté, mais aussi au stigmate de leur « indianité »- l'effet paradoxal de ces restitutions étant alors non pas de soulager mais bien de « renforcer » la douleur des « victimes » (p. 96). 
7 Dans la même veine, la contribution de C.Duterme («Honorer, commémorer, dédommager. État et société civile face aux victimes du conflit armé interne dans la région Ixil - Guatemala)» insiste sur le dédoublement du travail de prise en charge des dépouilles humaines et du deuil au sein de communautés rurales mayas. Contrastant avec celle de Sanford pour interroger le décalage entre, d'une part, des pratiques officielles et normalisées dans la droite ligne de l'accord d'Oslo ${ }^{3}$, et, d'autre part, une prise en charge plus intime, locale, de ces processus, elle révèle ainsi tout le travail - pragmatique, rituel, « officieux » - de ré-humanisation de morts retrouvés dans des états physiques difficilement soutenables, fondé sur l'élaboration ad hoc de pratiques de réassemblage, de ré-habillement et, plus généralement, de ré-humanisation de ces restes humains démembrés, lacunaires. Son analyse montre comment c'est là que se tisse, dans l'interaction entre les familles et certains experts médicaux, le retour à la « dignité » des morts (plutôt, par exemple, que dans des réparations économiques que les populations acceptent parfois, comme au Pérou, à leurs corps défendant et par nécessité matérielle).

Pour les groupes concernés, (re)trouver ces défunts, c'est en effet devoir, tant pour soi que pour eux, les "réparer", leur redonner une place qui fait sens et tenter de les transformer en de «bons morts " pour la communauté. Nul doute, comme le disent en substance les auteurs, que "déranger » ces disparus en les faisant revenir par un biais éminemment matériel - leurs restes physiques morcelés-, c'est aussi, sur un plan anthropologique, encourir le risque de déranger l'ordre du monde, lequel repose en partie sur une disjonction entre les vivants et les morts (p.15, p.129) et engage des idéologies et des imaginaires variables, parfois remodelés, de la mort violente.

9 Ainsi, A.-M. Losonczy nous explique dans une contribution au titre évocateur - « Retours assassins. Violence armée, suicide et exhumation dans l'économie de la mort des Emberá Katío (Chocó, Antioquia, Colombie)»- que l'attitude déroutante, fuyante que les Emberá semblent adopter lors des excavations de charniers désormais usuelles dans les politiques de réparation colombiennes, se comprend au regard de la « communauté de silence» qui soude le groupe à l'égard de certaines expériences. Elle prend sens à la lumière d'une théorie complexe de la personne qui fait des victimes des exactions passées - dont le retour peut être ardemment désiré, quoiqu'éminemment craint - des morts dangereux, soit des figures d'une "malemort " revisitée, dont la réapparition s'avère menaçante pour la survie des vivants, et incontrôlable par les voies rituelles chamaniques ordinaires.

C'est aussi de figures de "fantômes" habitant des corps meurtris dont nous parle, avec une sensibilité remarquable, l'article de D. Delacroix («Sortir de prison: revenir de la mort? Marquage du corps et de l'âme à travers l'expérience carcérale au Pérou»). Scrutant les interstices des actuelles procédures officielles d'indemnisation, l'auteure envisage les figures intermédiaires d'« ex-prisonniers» - des hommes - enrôlés de force dans le conflit, à la fois bourreaux et victimes. Brisés par l'expérience carcérale qui a fait d'eux des « âmes errantes » dont le retour physique au village est aussi problématique pour eux que pour les communautés, c'est par le déploiement d'un vocabulaire vernaculaire distinct de celui collectivement mobilisé face aux ONG, ainsi que par leur investissement silencieux dans la construction d'une certaine mémoire des "victimes » qu'ils disent à leur tour la violence et le deuil de soi. Comme ailleurs, après des décennies de conflit, il serait en effet illusoire d'imaginer qu'un récit univoque, fondée sur la définition de catégories monolithiques occultant la complexité des expériences de la guerre (p. 178), puisse suffire à les exprimer. 
11 Pris dans son ensemble ainsi que dans le détail des contributions, Retour des corps déploie donc une approche anthropologique du contemporain visant à repenser l'événement violent (les crimes de masse autant que la pratique de l'exhumation elle-même) dans ses déterminants historico-politiques tout en l'appuyant - et c'est là l'un de ses principaux apports - sur une réflexion de fond sur les sociétés et/ou les groupes considérés, ressaisis dans des matrices sociales et culturelles de longue durée, au carrefour de l'articulation entre "structure " et "conjoncture» (bien que ces termes ne soient pas directement mobilisés). Mettant en perspective, par des contextualisations précises et des enquêtes de terrain fouillées, l'une des déclinaisons les plus complexes de la globalisation culturelle ainsi que la fabrication (juridico-morale) et la mise en circulation conjointe de la valeur impliquée par cette mondialisation de la figure de la "victime", cet ouvrage collectif dialogue dès lors de façon féconde avec toute une littérature abordant des questions affines ${ }^{4}$. Outre qu'il accule son lecteur à des réflexions sur l'intrinsèque complexité de ces entreprises pacificatrices autant que sur la dialectique de la mémoire et de l'oubli - dialectique qui opère à l'échelle de la psyché individuelle et de la commémoration sociale -, le volume ouvre aussi sur des questionnements transversaux stimulants.

12 Le premier touche à la question des formes de ritualisation engendrées par le deuil extrême - dimension présente, en filigrane, de part en part de l'ouvrage. Ici, celles-ci semblent prendre place aux confins de formes de ritualité politique ou "religieuse » réinventées, ayant pour but de "guérir » le corps social. À la croisée de l'anthropologie du rituel et de l'anthropologie politique, se dessine alors une interrogation quant aux manières dont le rituel semble s'imposer comme une sorte de ressource naturelle mobilisée pour reconstruire, à défaut colmater, le collectif là où le monde se délite et le contrat social semble rompu; là où, en somme, la violence n'est autre que « rupture de l'intelligibilité du monde » (Fassin et Bensa 2002). La seconde, tout entière suggérée par le titre de cette recension, renvoie aux lignes novatrices que ce recueil tend à esquisser pour une possible ethnographie ou anthropologie du silence, dégagée du piège circulaire que lui tend la parole. De ce voyage éprouvant au pays de la violence, des morts et de leurs proches endeuillés, jaillit en effet tout ce que les groupes considérés mobilisent pour dire, revendiquer, rendre visible, mais aussi pour taire, ou dire autrement, l'expérience de la domination, de la perte et du deuil. Captant ces silences avec une finesse qui, parfois, force l'admiration, les auteurs font dès lors conjointement travailler intellect et émotions. L'une des questions lancinantes qui taraude le lecteur de bout en bout touche d'ailleurs aux conditions du travail empirique dans de tels contextes de douleur et de terreur sous-jacente, dimension sur laquelle on souhaiterait qu'une réflexion collective puisse se développer tant les affects semblent ici s'imposer comme l'un des ressorts de la production (et de la réception) d'une certaine compréhension anthropologique.

13 Dérangeant en même temps que passionnant, Retour des corps, parcours des âmes offre donc une première contribution américaniste (une fois n'est pas coutume, j'englobe ici la péninsule ibérique dans ses anciennes colonies) en langue française sur un sujet particulièrement difficile à aborder, ressaisi dans un questionnement foncièrement anthropologique sur le deuil et la reconfiguration du lien à la mort. Il a aussi pour immense mérite de contribuer à écrire et à faire connaître, au présent, une partie de l'histoire de « gens sans histoire » (Deschamps Chapeaux et Pérez de la Riva 1963) que les 
récits nationaux en cours d'élaboration, pris dans d'autres enjeux, peineront probablement toujours à raconter.

\section{BIBLIOGRAPHIE}

\section{BENSA Alban et Eric FASSIN}

2002 «Les sciences sociales face à l'événement », Terrain. Anthropologie \& sciences humaines, 38

(«Qu'est-ce qu'un événement?»), p. 5-20.

DESCHAMPS CHAPEAUX Pedro et Juan PÉREZ DE LA RIVA

1963 Contribución a la Historia de la gente sin historia, Editorial de Ciencias Sociales, La Havane.

GARIBIAN Sévane

2016 La mort du bourreau. Réflexions interdisciplinaires sur le cadavre des criminels de masse, Éditions Pétra (Les cadavres dans les génocides et les violences de masse), Paris.

\section{NOTES}

1. À l'exception des deux articles d'ouverture (F. Ferrándiz Martín, V. Sanford), les textes sont tous des inédits.

2. Créée par l'anthropologue E. Anstett et l'historien J.-M. Dreyfus, cette collection pluridisciplinaire, à l'édition sans fioriture mais au travail éditorial impeccable, augure de jeux de résonance féconds ; elle a depuis publié un troisième volume consacré à « la mort du bourreau » (Garibian 2016).

3. Accord signé en juin 1994 qui marque la création de la Commission pour l'éclaircissement historique au Guatemala.

4. Je pense en particulier aux travaux de D. Fassin sur la «condition victimaire » et la " raison humanitaire ", à ceux de la politiste S. Lefranc sur les processus de la « justice transitionnelle» ou encore à ceux d'E. Claverie et d'E. Anstett, tous régulièrement convoqués, au milieu de références hispanophones ou des réflexions plus anciennes, par exemple, de Primo Levi.

\section{AUTEURS}

\section{EMMA GOBIN}

Université Paris 8 Vincennes - Saint-Denis, LAVUE (UMR CNRS 7218, équipe AUS), « Mobilités, gouvernances et ressources dans le bassin méso-américain » (LMI MESO - IRD) 